\title{
Duality in the Azbel-Hofstadter problem and the two-dimensional d-wave superconductivity with a magnetic field
}

\author{
Y. Morita ${ }^{1}$ and Y. Hatsugai ${ }^{1,2}$ \\ Department of Applied Physics, University of Tokyo, 7-3-1 Hongo Bunkyo-ku, Tokyo 113-8656, Japan ${ }^{1}$ \\ PRESTO, Japan Science and Technology Corporation ${ }^{2}$
}

(October 30, 2018)

A single-parameter family of lattice-fermion model is constructed. It is a deformation of the Azbel-Hofstadter problem by a parameter $h=\Delta / t$ (quantum parameter). A topological number is attached to each energy band. A duality between the classical limit $(h=+0)$ and the quantum limit $(h=1)$ is revealed in the energy spectrum and the topological number.

The model has a close relation to the two-dimensional dwave superconductivity with a magnetic field. Making use of the duality and a topological argument, we shed light on how the quasiparticles with a magnetic field behave especially in the quantum limit.

Two-dimensional Dirac fermions with a gauge field are of current interest, e.g., in the context of the vortex state in a two-dimensional d-wave superconductivity |1 3. In our study, Dirac fermions with a gauge field are realized on a two-dimensional lattice. It is a single-parameter deformation of the Azbel-Hofstadter problem [4,5]. In this paper, the parameter $h$ is called quantum parameter [6]. A topological number is assigned for each energy band [7 9]. As the quantum parameter is varied continuously from the classical limit $(h=+0)$ to the quantum limit $(h=1)$, the energy spectrum is reconstructed through the change of each topological number (plateau transition [9 11]). Although the two limits are not connected adiabatically, we found that there is a duality between the classical and the quantum regime.

The model has a close relation to the two-dimensional d-wave superconductivity with a magnetic field. Applying the duality and a topological argument, we provide insights into the quasiparticle spectrum. In the quantum limit, interference effects become relevant especially at zero energy [3]. The existence of edge states is discussed as well. It reflects a non-trivial topology of each energy band [9, 12].

Let us define a key Hamiltonian in our paper, which is a single-parameter family of lattice fermion model. It is a deformation of the Azbel-Hofstadter problem. The Hamiltonian is $\mathcal{H}=\sum_{l, m} \mathbf{c}_{l}^{\dagger} \mathcal{H}_{l m} \mathbf{c}_{m}$ with

$$
\mathcal{H}_{l m}=e^{i A_{l m}}\left(\begin{array}{cc}
t_{l m}^{0} & \Delta_{l m}^{0} \\
\Delta_{m l}^{0} & -t_{m l}^{0}
\end{array}\right)
$$

$\mathbf{c}_{n}^{\dagger}=\left(c_{n \uparrow}^{\dagger} c_{n \downarrow}^{\dagger}\right), \mathbf{c}_{n}=\left(\begin{array}{c}c_{n \uparrow} \\ c_{n \downarrow}\end{array}\right), A_{l m}=-A_{m l} \in \mathbf{R}$ and $\sum_{\square} A_{l m} / 2 \pi=\phi=p / q(p$ and $q$ are coprime integers and the summation runs over four links around a plaquette). Here $l, m \in \mathbf{Z}^{2}, t_{m \pm(1,0), m}^{0}=t_{m \pm(0,1), m}^{0}=t, \Delta_{m \pm(1,0), m}^{0}=$ $-\Delta_{m \pm(0,1), m}^{0}=\Delta(t, \Delta \in \mathbf{R})$ and the other matrix elements are zero. $t$ is set to be a unit energy and a relevant parameter, quantum parameter, is defined by $h=\Delta / t$. The relation of this model to the two-dimensional d-wave superconductivity with a magnetic field is discussed later. In the classical limit $h=+0$, this model decouples to two essentially equivalent Hamiltonians. It is the AzbelHofstadter Hamiltonian $\sum_{l, m} c_{l}^{\dagger} e^{i A_{l m}} t_{l m}^{0} c_{m}$. The energy spectrum at $\phi=0$ is given by $E= \pm \sqrt{A(k)^{2}+|B(k)|^{2}}$ where $A(k)=2 t\left(\cos k_{x}+\cos k_{y}\right), B(k)=2 \Delta\left(\cos k_{x}-\right.$ $\left.\cos k_{y}\right)$ and $k \in[-\pi, \pi] \times[-\pi, \pi]$. The upper and lower bands touch at four points $( \pm \pi / 2, \pm \pi / 2)$ in the Brillouin zone. The low-lying excitations around the gap-closing points are described by massless Dirac fermions.

One of the basic observables is a topological number for the $n$-th band, $\mathcal{C}_{n}[9]$. It is

$$
\mathcal{C}_{n}=\frac{1}{2 \pi i} \int d \mathbf{k} \hat{z} \cdot\left(\nabla \times \mathbf{A}_{n}\right), \mathbf{A}_{n}=\left\langle u_{n}(\mathbf{k})|\nabla| u_{n}(\mathbf{k})\right\rangle
$$

where $\nabla=\partial / \partial \mathbf{k}$ and $\left|u_{n}(\mathbf{k})\right\rangle$ is a Bloch vector of the $n$-th band. The integration $\int d \mathbf{k}$ runs over the Brillouin zone (torus). The non-zero topological number results in the existence of edge states. In order to see it, put the system on a cylinder and introduce a fictitious flux through the cylinder (it is equivalent to a twist in the boundary condition) [13]. The edge states move from one boundary to the other as the fictitious flux quanta $h c / e$ is added adiabatically. The number of carried edge states coincides with the summation of topological numbers below the Fermi energy [9, 12]. Due to the topological stability, a singularity necessarily occurs with the change of the topological number (plateau transition [9 11]). The singularity is identified with an energy-gap closing on some points in the Brillouin zone.

In Figs. 1-3, the energy spectra are shown. As $h$ is varied continuously from the classical limit $(h=+0)$ to the quantum limit $(h=1)$, the energy spectrum is reconstructed through the plateau transitions. Although the two limits are not connected adiabatically, a main feature in the data is that there is a symmetry between the classical and the quantum regime. It leads to a claim that 
' there is a faithful correspondence between $\phi=(1 / 2+$ $p / q)$ in the classical limit $h=+0$ and $\phi=p / q$ in the quantum limit $h=1$ '.

We call this phenomena duality. As discussed above, this model reduces to a doubled Azbel-Hofstader problem in the classical limit $h=+0$. The duality is an analogue of a statistical transmutaion (composite fermion picture) in the fractional quantum Hall effect [14]. In the composite fermion picture, a locally attached flux to each fermion is replaced with a global uniform flux. In our case, pairing effects (off-diagonal order) play the role of shifting a flux globally. It is reminiscent of a symmetry between the d-wave pairing and the $\pi$-flux, in other words, the $S U(2)$ symmetry [15]. Here some comments are in order. At $\phi=0$ and $1 / 2$, we proved analytically that the claim is exact. Moreover, as shown in Figs. 1 and 3 , topological numbers are consistent with the claim.

As an application of the duality, let us study the twodimensional $d$-wave pairing model with a magnetic field especially in the quantum limit $(h=1)$. The pairing model is $H=\sum_{l, m} \mathbf{c}_{l}^{\dagger} H_{l m} \mathbf{c}_{m}$ with

$$
H_{l m}=\left(\begin{array}{cc}
t_{l m} & \Delta_{l m} \\
\Delta_{m l}^{*} & -t_{m l}
\end{array}\right)
$$

Under the unitary transformation $c_{n \uparrow} \rightarrow d_{n \uparrow}, \quad c_{n \downarrow} \rightarrow d_{n \downarrow}^{\dagger}$ (for $\forall n$ ), it becomes $H=\sum_{l, m}\left[d_{l \uparrow}^{\dagger} t_{l m} d_{m \uparrow}+d_{l \downarrow}^{\dagger} t_{l m} d_{m \downarrow}+\right.$ $d_{l \uparrow}^{\dagger} \Delta_{l m} d_{m \downarrow}^{\dagger}+d_{m \downarrow} \Delta_{l m}^{*} d_{l \uparrow}$. It is equivalent to the Bogoliubov-de Gennes Hamiltonian for the singlet superconductivity. Here $t_{l m}^{*}=t_{m l}$ (hermiticity) and $\Delta_{l m}=$ $\Delta_{m l}(\mathrm{SU}(2)$ symmetry) are imposed as well. It satisfies a relation $-\left(\sigma_{y} H_{l m} \sigma_{y}\right)^{*}=H_{l m}$, which results in a particle-hole symmetry in the energy spectrum. The twodimensional $d$-wave pairing model with a magnetic field is defined by the pairing model (2) with

$$
\begin{aligned}
t_{l m} & =\exp \left(i A_{l m}\right) t_{l m}^{0}, \\
\Delta_{l m} & =\exp \left(i \varphi_{l m}\right) \Delta_{l m}^{0}
\end{aligned}
$$

where $A_{l m}=-A_{m l}, \varphi_{l m}=\left(\varphi_{l}+\varphi_{m}\right) / 2 \in \mathbf{R}$ so that $t_{l m}^{*}=$ $t_{m l}$ (hermiticity) and $\Delta_{l m}=\Delta_{m l}$ (SU(2) symmetry) are satisfied [1,3]. Performing a gauge transformation [1]

$$
\mathbf{c}_{n} \rightarrow\left(\begin{array}{cc}
e^{i \varphi_{n}} & 0 \\
0 & 1
\end{array}\right) \mathbf{c}_{n}
$$

we obtain

$$
H_{l m}=e^{-i A_{l m}}\left(\begin{array}{cc}
t_{l m}^{0} e^{-2 i v_{l m}} & \Delta_{l m}^{0} e^{-i v_{l m}} \\
\Delta_{m l}^{0} e^{-i v_{l m}} & -t_{m l}^{0}
\end{array}\right)
$$

where $v_{l m}=\left(\varphi_{l}-\varphi_{m}\right) / 2-A_{l m}$. It is a lattice realization of the Hamiltonian discussed in ref. [1] and $v_{l m}$ corresponds to the superfluid velocity. In the case $v_{l m}=0$, it reduces to the Hamiltonian (1) and the quasiparticle spectrum consists of 'Landau levels' $E= \pm \omega_{H} \sqrt{n}(n \in \mathbf{N})$ in the continuum limit [1].
In the following, we set a period for $A_{l m}$ and $v_{l m}$ to be $l_{x} \times l_{y}$. Here we emphasize that, in the context of superconductivity, $A_{l m}$ and $v_{l m}$ are determined in a selfconsistent way. Moreover, the spatial variation of $\left|\Delta_{l m}\right|$ plays a crucial role especially near a vortex core. In our study, however, $A_{l m}$ and $v_{l m}$ are treated as adjustable parameters and focus is put on the duality or topological arguments which do not depend on the detail of the potential.

In Fig.4, an example of the density of states (DoS) is shown for the $d$-wave pairing model with a magnetic field in the quantum limit $(h=1)$. Although the weakfield regime $(\phi \sim 0)$ is relevant to reality, we show the case $\phi=1 / 5$ for clarity. In the weak-field regime, the number of energy bands increases but the following arguments are robust.

As the system at $\phi=p / q$ approaches the quantum regime $(h \sim 1)$, the quasiparticle spectrum becomes close to that of the Azbel-Hofstadter problem at $\phi=(1 / 2+$ $p / q)$. The Landau levels are mixed due to lattice effects and $v_{l m}$. It causes a singularity at zero energy [3] and the broadening of each level ('Landau bands'). It is due to quantum interference effects through spatially varying potentials. It is an analogue of the vanishing DoS at zero energy in random Dirac fermions 16 18. In the case of $\phi=p / q(q=$ odd $)$, it is a natural consequence of the duality. In other words, it can be interpreted by the fact that there exists a singularity at zero energy i.e. $\rho(E=0)=0$ in the Azbel-Hofstadter problem at $\phi=(1 / 2+p / q)$. It is also to be noted that, apart from the singularity and the broadening, the energy spectrum of the Azbel-Hofstadter problem at $\phi=(1 / 2+p / q)(p=$ $1, q \gg 1)$ is $\sim \pm \omega_{H} \sqrt{n}$ near the band center and $\sim \pm(\omega n+$ $C)$ near the band edge $(n \in \mathbf{N})$.

Now put the system on a cylinder (periodic in the $y$ direction and open in the $x$ direction) and let us study the basic properties of edge states in the d-wave pairing model with a magnetic field. The Schrödinger equation for the Hamiltonian (2) is

$$
\sum_{j}\left(\begin{array}{cc}
t_{i j} & \Delta_{i j} \\
\Delta_{j i}^{*} & -t_{j i}^{*}
\end{array}\right)\left(\begin{array}{l}
u_{j} \\
v_{j}
\end{array}\right)=E\left(\begin{array}{l}
u_{i} \\
v_{i}
\end{array}\right) .
$$

Decompose all the sites $\mathcal{N}$ into two sublattices $A$ and $B$ $(A \cup B=\mathcal{N}$ and $A \cap B=\phi)$ where $t_{i j}$ and $\Delta_{i j}$ connecting the same sublattice are zero. Define $\bar{w}_{k}$ by $\bar{w}_{k}=+w_{k}$ $(k \in A)$ and $\bar{w}_{k}=-w_{k}(k \in B)$. Then it follows from the $\mathrm{SU}(2)$ symmetry that

$$
\sum_{j}\left(\begin{array}{cc}
t_{i j} & \Delta_{i j} \\
\Delta_{j i}^{*} & -t_{j i}^{*}
\end{array}\right)\left(\begin{array}{c}
-\bar{v}_{j}^{*} \\
\bar{u}_{j}^{*}
\end{array}\right)=E\left(\begin{array}{c}
-\bar{v}_{i}^{*} \\
\bar{u}_{i}^{*}
\end{array}\right) .
$$

It leads to the claim that, if an edge state exists, a paired (degenerate but linearly independent) edge state can be constructed which is localized in the same side [19.

Next we shall discuss the existence of edge states. At first, set $\phi=p / q, v_{l m}=0$ and focus on an energy gap with a non-zero topological number (see, for example, 
Fig.3. The duality implies that a topological number for a generic gap is non-zero even number in the quantum regime. It is consistent with the previous result [9]). As discussed above, a non-zero topological number leads to the existence of edge states in the gap. In other words, the existence of edge states is topologically stable. Next consider the case when $v_{l m}$ is finite. As the $v_{l m}$ is varied from zero to the finite value, the Landau bands are deformed and can be overlapped like a semimetal. We can, however, observe edge states due to the topological stability, when the plateau transition is absent in the deformation. In fact, see Fig.5. The existence of edge states can be confirmed in each energy gap. It reflects a non-trivial topology of each energy band. In the above discussion, we have employed a cylinder. It is possible to consider the same problem on a geometry with 'defects' (e.g. annulus). In the case, the states analogous to the edge states on a cylinder may be bound to the defects.

In summary, a single-parameter family of latticefermion model is constructed in two dimensions. The parameter $h=\Delta / t$ is called quantum parameter. A duality is revealed in the model between the classical limit $(h=+0)$ and the quantum limit $(h=1)$. Employing the duality and a topological argument, we provide insights into how the quasiparticles with a magnetic field behave especially in the quantum limit. A more detailed study including a self-consistent potential and the fluctuation is left as a future problem. It is crucial for the analysis of dynamical properties. As discussed in the context of the integer quantum Hall effect, when the potential is sufficiently strong, it can bring the system to a totally different state 20].

One of the authors (Y.M.) thanks H. Matsumura for valuable discussions. This work was supported in part by Grant-in-Aid from the Ministry of Education, Science and Culture of Japan. The computation has been partly done using the facilities of the Supercomputer Center, ISSP, University of Tokyo.

FIG. 1. $\phi=p / 127, h=\Delta / t=0$. An integer in an energy gap is the summation of topological numbers below the gap.

FIG. 2. $\phi=p / 127, h=\Delta / t=0.5$. An integer in an energy gap is the summation of topological numbers below the gap.

FIG. 3. $\phi=p / 127, h=\Delta / t=1$. An integer in an energy gap is the summation of topological numbers below the gap.

FIG. 4. An example of the DoS for the $d$-wave pairing model $(h=\Delta / t=1)$ with a magnetic field $(p=1, q=5$, $\phi=1 / 5)$. The spatial average and variance of $v_{l m}$ is 0 and 0.1 respectively. The period $l_{x} \times l_{y}$ is $5 \times 5$. The DoS for the $d$-wave pairing model $(h=1)$ without a magnetic field is also shown (broken line).
FIG. 5. Energy spectrum of the $d$-wave pairing model $(h=\Delta / t=1)$ with a magnetic field $(p=1, q=3, \phi=1 / 3)$ on a cylinder (the boundary is periodic in the $y$ direction and open in the $x$ direction). The spatial average and variance of $v_{l m}$ is 0 and 0.1 respectively. The period $l_{x} \times l_{y}$ is $30 \times 3$. The system is composed of $1 \times 100$ cells.

[1] P. W. Anderson, preprint (cond-mat/9812063).

[2] L. P. Gor'kov and J. R. Schrieffer, Phys. Rev. Lett. 80, 3360 (1998).

[3] L. Marinelli, B. I. Halperin and S. H. Simon, preprint (cond-mat/0001406) and references therein.

[4] M. Ya Azbel, Sov. Phys. JETP 19634 (1964).

[5] D. R. Hofstadter, Phys. Rev. B 14, 2239 (1976).

[6] Y. Morita, M. Kohmoto and K. Maki, Int. J. of Mod. Phys. B 12, 989 (1998).

[7] D. J. Thouless, M. Kohmoto, P. Nightingale, and M. den Nijs, Phys. Rev. Lett. 49, 405 (1982).

[8] Y. Hatsugai, J. Phys.: Condens. Matter 49, 2507 (1997).

[9] Y. Morita and Y. Hatsugai, Phys. Rev. B 62, 99 (2000).

[10] Y. Hatsugai and M. Kohmoto, Phys. Rev. B 42, 8282 (1990).

[11] M. Oshikawa, Phys. Rev. B 50, 17357 (1994).

[12] Y. Hatsugai, Phys. Rev. Lett. 71, 3697 (1993); Phys. Rev. B 48, 11851 (1993).

[13] R. B. Laughlin, Phys. Rev. B 23, 5632 (1981).

[14] J. K. Jain, Phys. Rev. B 41, 7653 (1990).

[15] I. Affleck, Z. Zou, T. Hsu and P. W. Anderson, Phys. Rev. B 38, 745 (1988).

[16] A. Ludwig, M. Fisher, R. Shankar and G. Grinstein, Phys. Rev. B 50, 7526 (1994).

[17] A. A. Nersesyan, A. M. Tsvelik and F. Wenger, Phys. Rev. Lett. 72, 2628 (1994).

[18] Y. Morita and Y. Hatsugai, Phys. Rev. Lett. 79, 3728 (1997); Phys. Rev. B 58, 6680 (1998).

[19] If the paired edge state is linearly dependent, $\left|u_{i}\right|^{2}+$ $\left|v_{i}\right|^{2}=0$ must hold for all sites. Since the state is not a null vector, it leads to a contradiction.

[20] Y. Hatsugai, K. Ishibashi and Y. Morita, Phys. Rev. Lett. 83, 2246 (1999); Y. Morita, K. Ishibashi and Y. Hatsugai, Phys. Rev. B 61, 15952 (2000). 


\section{Fig.1}

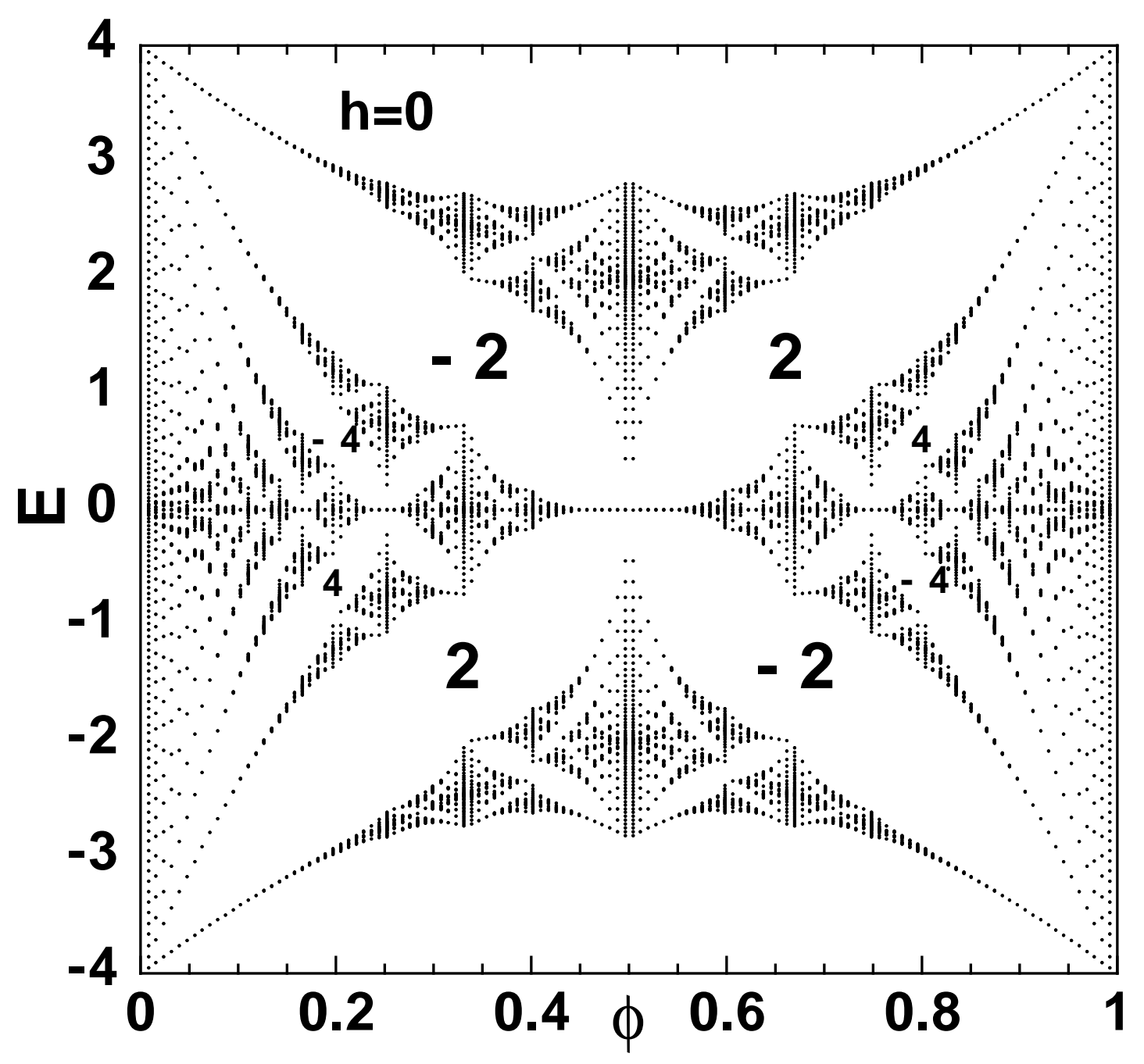


Fig.2

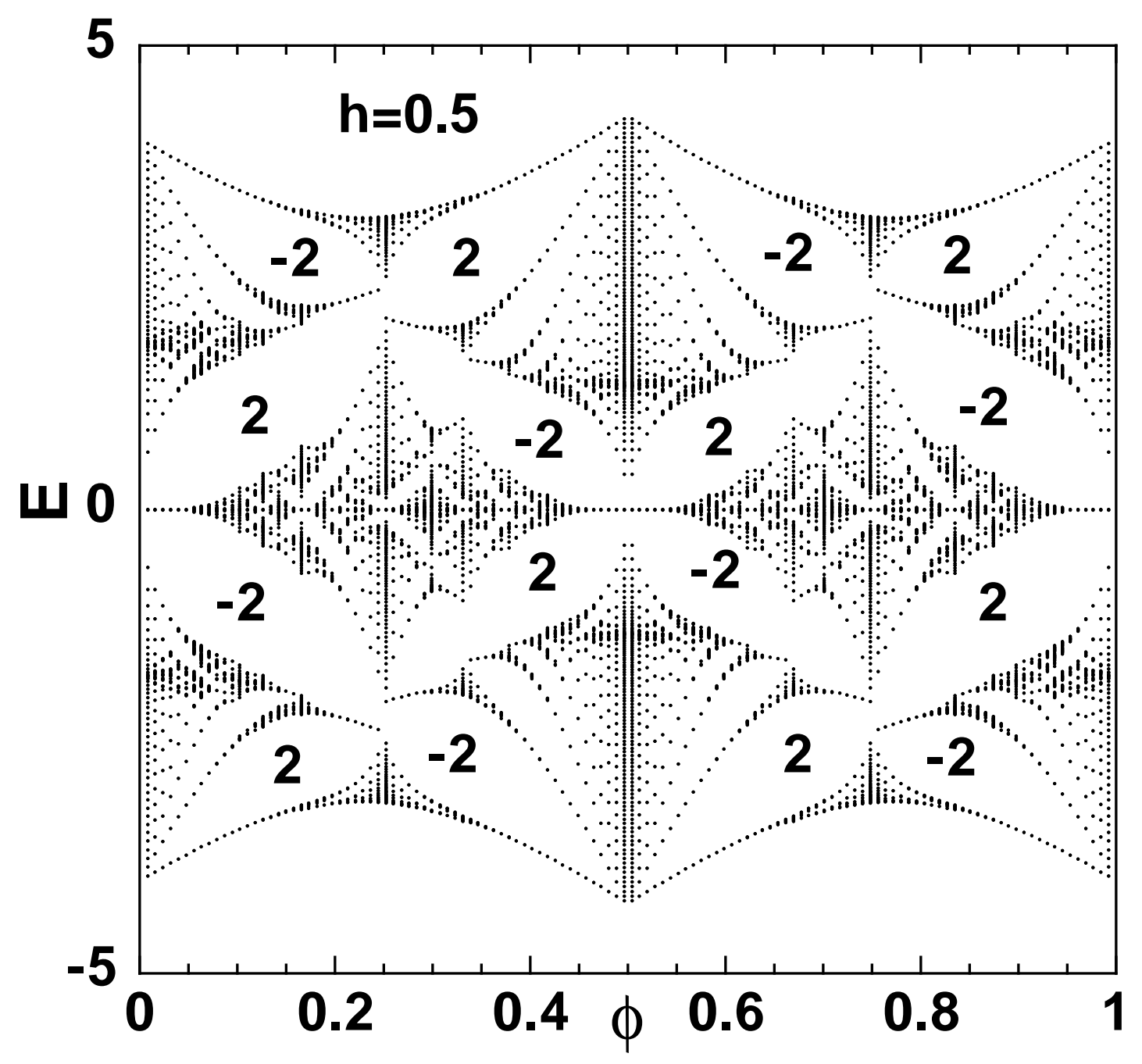




\section{Fig.3}

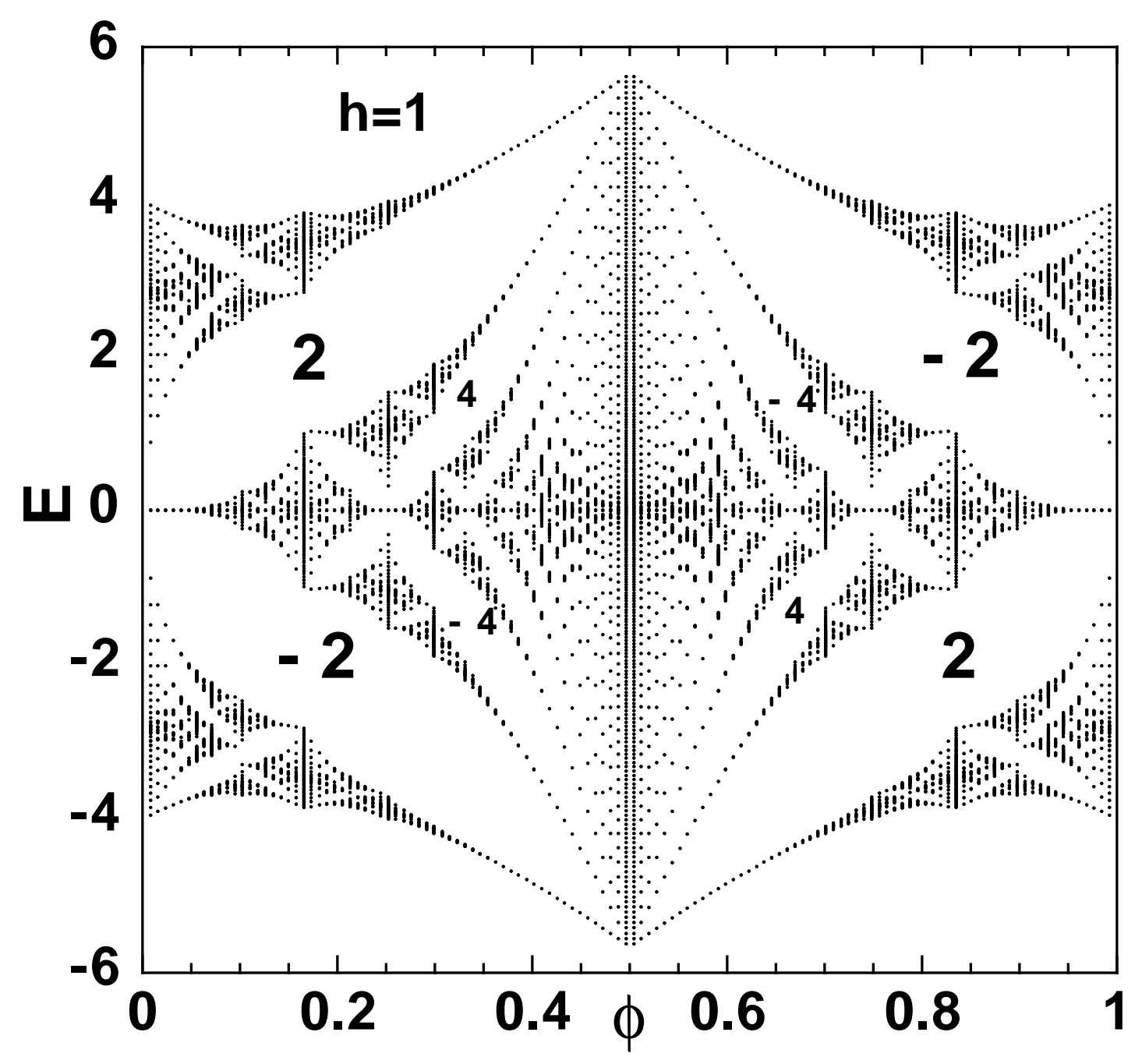


Fig.4






\section{Fig.5}

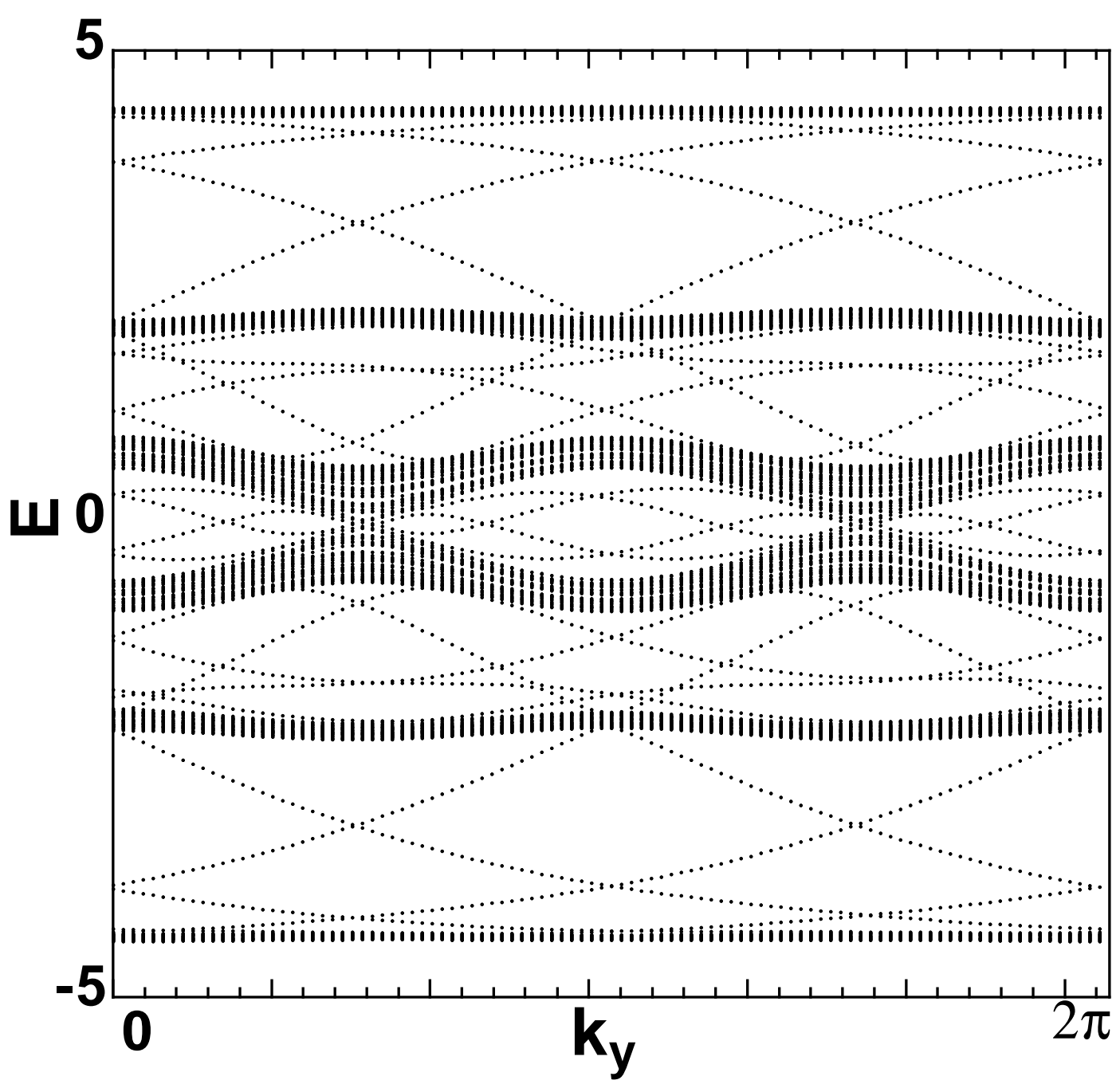

